\title{
Myelin oligodendrocyte glycoprotein antibodies in pathologically proven multiple sclerosis: frequency, stability and clinicopathologic correlations
}

\author{
SJ Pittock ${ }^{1}, M$ Reindl $^{2}$, S Achenbach ${ }^{5}, T$ Berger $^{2}, W$ Bruck $^{3}, F$ Konig $^{3}, Y_{\text {Morales }}$, \\ H Lassmann ${ }^{4}$, S Bryant 5 , SB Moore ${ }^{6}$, BM Keegan ${ }^{1}$ and CF Lucchinetti*1
}

\begin{abstract}
Controversy exists regarding the pathogenic or predictive role of anti-myelin oligodendrocyte glycoprotein (MOG) antibodies in patients with multiple sclerosis (MS). Four immunopathological patterns (IP) have been recognized in early active MS lesions, suggesting heterogeneous pathogenic mechanisms. Whether MOG antibodies contribute to this pathological heterogeneity and potentially serve as biomarkers to identify specific pathological patterns is unknown. Here we report the frequencies of antibodies to human recombinant MOG (identified by Western blot and enzymelinked immunoabsorbent assay (ELISA)) in patients with pathologically proven demyelinating disease, and investigate whether antibody status is associated with clinical course, HLA-DR2genotype, IP or treatment response to plasmapheresis. The biopsy cohort consisted of 72 patients: 12 pattern I, 43 pattern II and 17 pattern III. No association was found between MOG antibody status and conversion to clinically definite MS, DR-2 status, IP or response to plasmapheresis. There was poor agreement between Western blot and ELISA (kappa $=0.07$ for MOG IgM). Fluctuations in antibody seropositivity were seen for 3/4 patients tested serially by Western blot. This study does not support a pathologic pattern-specific role for MOG-antibodies. Variable MOG-antibody status on serial measurements, coupled with the lack of Western blot and ELISA correlations, raises concern regarding the use of MOG-antibody as an MS biomarker and underscores the need for methodological consensus. Multiple Sclerosis 2007; 13: 7-16. http://msj.sagepub.com
\end{abstract}

Key words: ELISA; immunological patterns; MOG antibodies; multiple sclerosis; Western blot

\section{Introduction}

There is controversy regarding the pathogenic or prognostic role of anti-myelin oligodendrocyte glycoprotein (MOG) antibodies in patients with multiple sclerosis (MS) or isolated demyelinating syndromes (IDS). Although MOG antibodies have been described in MS lesions, reported frequencies of these antibodies in MS patients vary between studies and MOG antibodies are present in both patients with non-inflammatory neurological diseases and in normal controls [1-11]. The discre- pancies between studies raise concerns regarding the sensitivity and specificity of these antibodies for MS. Investigators cite technical differences in antigen preparation and antibody determination as possible explanations for these discrepancies, further highlighting the lack of methodological consensus for MOG antibody detection. International multicentre validation experiments are underway in order to address these methodological differences. A recent study suggested MOG IgM antibodies are associated with increased disease activity in MS, and may also be a predictor of early

\footnotetext{
${ }^{1}$ Department of Neurology, Mayo Clinic, Rochester, MN, USA

2 Clinical Department of Neurology, Innsbruck Medical University, Innsbruck, Austria

${ }^{3}$ Department of Neuropathology, University of Göttingen, Göttingen, Germany

${ }^{4}$ Brain Research Institute, University of Vienna, Vienna, Austria

5 Department of Biostatistics, Mayo Clinic, Rochester, MN, USA

${ }^{6}$ Department of Laboratory Medicine and Pathology, Mayo Clinic, Rochester, MN, USA
}

Author for correspondence: Claudia F Lucchinetti MD, Department of Neurology, Mayo Clinic, 200 First Street SW, Rochester, MN, USA. E-mail: lucchinetti.claudia@mayo.edu

Received 28 February 2006; accepted 11 June 2006 
conversion to definite MS in patients with a clinically isolated syndrome [12]. A subsequent study could not confirm this finding [13]. Despite these variable results, there are increasing numbers of requests by neurologists to test patients for the presence of anti-MOG antibodies.

Four immunopathological patterns (IPs) (I: T-cell/macrophage associated; II: antibody/complement associated; III: distal oligodendrogliopathy; and IV: oligodendrocyte degeneration in the periplaque white matter) have been described in autopsied and biopsied early active MS lesions, suggesting heterogeneous pathogenic mechanisms [14-16]. It is uncertain whether MOG antibodies are found preferentially in any of the pathological patterns (eg, in antibody-associated pattern II) or whether their presence is associated with therapeutic response, thereby supporting a pathogenic role for MOG. Keegan et al. reported a significant association between response to therapeutic plasma exchange (TPE) and MS pathological pattern. Patients with pattern II pathology were more likely to improve with TPE than patients with patterns I or III [17].

We determined, in a blinded fashion, the frequency of MOG antibodies in sera from patients with biopsy-proven demyelinating disease using two methodologies (enzyme-linked immunosorbent assay (ELISA) and Western blot), and examined MOG-antibody status with respect to clinical course, HLA DR-2 genotype, IP, and treatment response to TPE. Lastly, we assessed agreement between ELISA and Western blot results for MOG antibodies, and whether antibody seronegativity or positivity persisted in serially tested MS patients.

\section{Methods}

This study was approved by the Mayo Clinic Institutional Review Board (IRB \# 2067-99). The Multiple Sclerosis Lesion Project (MSLP) is an international collaborative effort to study the pathologic, clinical and radiologic correlates of the MS lesion. The MSLP is based on previous observations describing four IPs of pathology in early MS lesions with interindividual heterogeneity, but intraindividual homogeneity in the specific pattern identified. These findings suggest different pathogenic mechanisms may contribute to tissue injury in early MS. This may have treatment implications for clinical practice, as distinct patterns may require distinct therapeutic strategies [18].

One hundred and thirty patients with pathologically confirmed inflammatory demyelinating disease are currently being followed prospectively by the MSLP. We included 72 patients who had stored sera available for MOG antibody testing and who had biopsy-proven demyelinating disease classifiable into one of the previously reported four patterns. HLA typing was performed on 69 of these 72 patients. Patients with biopsies showing perivenular demyelination consistent with acute disseminated encephalomyelitis (ADEM) were excluded (as part of the MSLP criteria) [19].

Anti-MOG-Ig (rhMOG 1-125) antibodies were analysed by Western blot as previously described, with the following modifications: [4,7] $1 \mu \mathrm{g}$ of rhMOG-Ig was loaded per lane and separated in $10 \%$ Bis-Tris (NuPage) sodium dodecyl sulphate (SDS)-polyacrylamide gels (Invitrogen, Carlsbad, USA). Separated proteins were electrotransferred to nitrocellulose membranes (Hybond-C, Amersham, UK). Efficiency of transfer was monitored by the use of a prestained low range SDS-PAGE standard (BioRad, Hercules, USA) and by staining of the filters with Ponceau S (Sigma, St. Louis, USA) after transfer. Blots were then blocked with $2 \%$ milk powder in PBS containing 0.05\% Tween-20. The blots were then dried, cut into $2-\mathrm{mm}$ nitrocellulose strips with a membrane cutter (Invitrogen) and probed overnight at $4{ }^{\circ} \mathrm{C}$ with diluted human serum (for IgG 1:1000, for IgM 1:500; all diluted in 2\% milk powder in PBS containing 0.05\% Tween-20). Strips were then washed three times with PBS containing $0.05 \%$ Tween-20 and incubated either with alkaline-phosphatase-conjugated anti-human IgG (dilution, 1:5000; JGH-055003, Jackson, USA) or alkaline-phosphatase-conjugated anti-human IgM (dilution, 1:5000; JGH-055043, Jackson, USA) for $1 \mathrm{~h}$ at room temperature. After washing, bound antibodies were detected by p-nitro-blue-tetrazolium chloride and 5-bromo-4-chloro-3-indolylphosphate (both, Roche Molecular Diagnostics, Mannheim, Germany). The strips were then washed with distilled water, dried and immunoreactivity of the serum samples was assessed semiquantitatively by three different blinded investigators. Representative immunoblots of control antibodies, control sera and MS sera are shown in Figure 1. A serum sample was considered to be positive if the immunoreactivity was equal to or stronger than that of the control sample. For controls we used a monoclonal antibody to MOG (8.18-C5) to identify the correct position of MOG as well as a human control serum to check the interassay variation.

For ELISA testing, rhMOG-Ig was expressed in $E$. coli and purified by nickel-chelate affinity chromatography on chelating Sepharose Fast Flow (Pharmacia, Uppsala, Sweden) using a novel single-step purification and refolding procedure [20]. ELISA was performed using 96-well microtitre plates (Nunc-Immuno Maxisorp, Nunc, USA) coated with $100 \mu \mathrm{L}$ of a $5 \mu \mathrm{g} / \mathrm{mL}$ rhMOG $^{\text {Igd }}$ solution in PBS overnight at $4^{\circ} \mathrm{C}(0.5 \mu \mathrm{g} /$ well $)$. Plates were then 


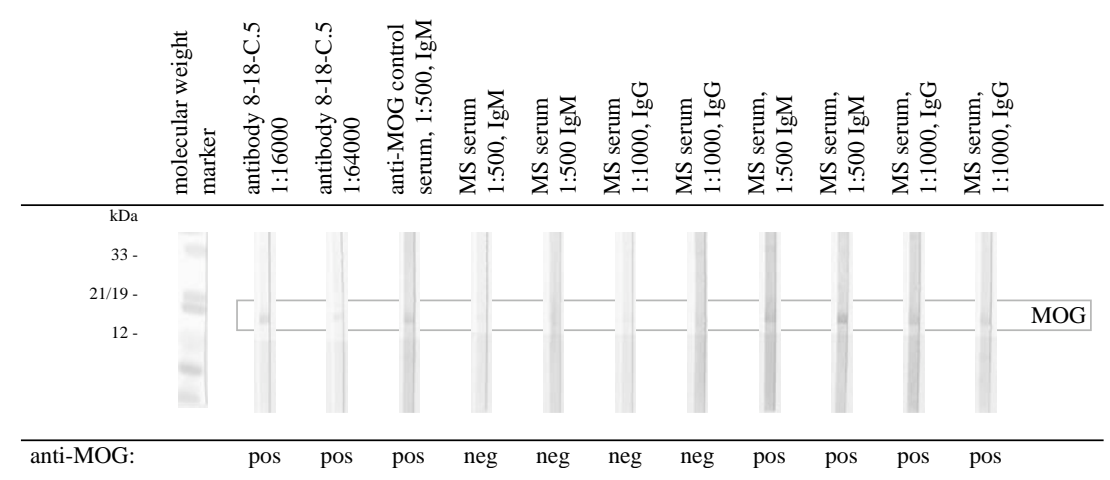

Figure 1 Representative raw immunoblot data. Immunoblots were incubated with monoclonal antibody to MOG (8-18-C.5), a human control serum positive for anti-MOG antibodies and MS serum samples. The correct position of MOG was identified by the monoclonal antibody 8-18-C.5 and is indicated in the figure.

blocked with $10 \%$ fetal calf serum (FCS; Invitrogen) in PBS (PBS-FCS) for $1 \mathrm{~h}$ at room temperature and washed four times with PBS-0.05\% Tween-20 (PBS-T). Then $100 \mu \mathrm{L}$ of the serum samples diluted 1:100 (for IgG) or 1:250 (for IgM) in PBS-FCS were added and incubated for $1 \mathrm{~h}$ at room temperature with gentle shaking. Plates were washed and $100 \mu \mathrm{L}$ of peroxidase-conjugated goat anti-human IgM (1:5000) or IgG (1:10000) secondary antibody (Dako) in PBS-FCS were added for $1 \mathrm{~h}$ at room temperature with gentle shaking. Plates were washed, and specific antibody binding was visualized by the addition of $100 \mu \mathrm{L}$ of tetramethylbenzidine (TMB) liquid substrate (Sigma). After $20 \mathrm{~min}$ the reaction was stopped with $50 \mu \mathrm{L} 1 \mathrm{M} \mathrm{H}_{2} \mathrm{SO}_{4}$ and the plates were read at $492 \mathrm{~nm}$. Controls wells were incubated only with secondary antibodies. All data were corrected by subtraction of these background values. All samples were analysed in duplicate and three human control sera were used to check the interassay variation on all ELISA plates. The interand intra-assay variations were $<15 \%$. From 188 age- and sex-matched healthy controls from Innsbruck, Austria, we determined a 'cut-off' value for anti-MOG IgG (0.6 OD units) and IgM (1.3 OD units). The frequencies of anti-MOG antibodies in healthy controls $(n=188)$ were $10 \%$ for IgM and $12 \%$ for IgG, in clinically definite (CD) MS patients from Innsbruck, Austria $(n=202), 23 \%$ for IgM and $35 \%$ for IgG.

HLA-DR genotyping was assessed by PCR-SSP methods as previously described [21]. Follow-up and Expanded Disability Status Scale (EDSS) scores were ascertained by interview and examination, telephone interview and chart review [22]. The neuropathological techniques have been previously described, and lesions were immunopathologically classified according to published criteria $[14,15]$. We recorded demographic data, dates of symptom onset, index attack (attack leading to biopsy) and blood draws, and concomitant use of immunomo- dulatory or immunosuppressive treatments. The course of MS was categorized as relapsing-remitting (RRMS), secondary progressive (SPMS) or progressive relapsing (PR). Definite or probable MS by Poser and/or McDonald criteria were used to classify patients $[23,24]$. The course was categorized as uncertain when it was unclear if an episode represented an attack (eg, seizure). Those with only a single attack who did not fulfil Poser criteria for probable MS were considered IDS.

Standard descriptive statistics were used to summarize characteristics of the study patients, including medians and interquartile ranges (IQRs) for the continuous variables, and counts and percentages for the categorical variables. Continuous variables were compared between cohorts using Wilcoxon Rank Sum tests, and categorical variables using Fisher's exact tests. Kappa test was used to assess the agreement between Western blot and ELISA results. Statistical significance was defined as $P \leq 0.05$.

\section{Results}

\section{Clinical characteristics and MOG antibody status of MS cohort}

The clinical characteristics and antibody status of the 72 patients with pathologically proven demyelinating disease whose sera were tested for antiMOG antibodies (by Western blot and ELISA) are shown in Table 1. Sixty-five patients fulfilled Poser or McDonald criteria for clinically definite $(n=51)$, laboratory-supported definite $(n=6)$ or probable $(n=8)$ MS. Seven additional patients had an isolated attack (IDS after a median follow up of 7.5 years). Median delay from disease onset (for all 72 patients) to blood draw for antibody testing was 4.4 years (IQR 2.1-8.8). 
Table 1 Clinical characteristics of biopsied cohort

\begin{tabular}{|c|c|c|c|c|c|c|c|c|c|}
\hline \multirow[b]{3}{*}{ Antibody presence } & \multicolumn{5}{|c|}{ Western blot } & \multicolumn{4}{|c|}{ ELISA } \\
\hline & \multirow{2}{*}{ Total $(n=72)$} & \multicolumn{2}{|c|}{ MOG IgM } & \multicolumn{2}{|l|}{ MOG IgG } & \multicolumn{2}{|c|}{ MOG IgM } & \multicolumn{2}{|c|}{ MOG IgG } \\
\hline & & Positive & Negative & Positive & Negative & $\begin{array}{l}\text { Positive } \\
(>1.3)\end{array}$ & $\begin{array}{l}\text { Negative } \\
(<1.3)\end{array}$ & $\begin{array}{l}\text { Positive } \\
(>0.6)\end{array}$ & $\begin{array}{l}\text { Negative } \\
(<0.6)\end{array}$ \\
\hline \multicolumn{10}{|l|}{ Clinical course, $n(\%)$} \\
\hline $\begin{array}{l}\text { IDS at last follow-up, } \\
n(\%)\end{array}$ & 7 & $3(43)$ & $4(57)$ & $2(29)$ & $5(71)$ & $0(0)$ & $7(100)$ & $1(14)$ & $6(86)$ \\
\hline $\begin{array}{l}\text { MS at last follow-up, } \\
n(\%)\end{array}$ & 65 & $35(54)$ & $30(46)$ & $17(26)$ & $48(74)$ & $3(5)$ & $62(95)$ & $15(23)$ & $50(77)$ \\
\hline \multicolumn{10}{|c|}{ Therapies used within 6 months of antibody testing } \\
\hline $\begin{array}{l}\text { Immunomodulatory } \\
\text { drugs }^{*}, n(\%) \text { yes }\end{array}$ & $31(43)$ & $18(47)$ & $13(38)$ & $10(53)$ & $21(40)$ & $1(33)$ & $30(43)$ & $8(50)$ & $23(41)$ \\
\hline $\begin{array}{l}\text { Immunosuppressant } \\
\text { drugs }^{*}, n(\%) \text { yes }\end{array}$ & $4(6)$ & $3(8)$ & $1(3)$ & $1(5)$ & $3(6)$ & $0(0)$ & $4(6)$ & $1(6)$ & $3(5)$ \\
\hline $\begin{array}{l}\text { Long-term steroids, } \\
n(\%) \text { yes }\end{array}$ & $4(6)$ & $3(8)$ & $1(3)$ & $1(5)$ & $3(6)$ & $0(0)$ & $4(6)$ & $2(13)$ & $2(4)$ \\
\hline \multicolumn{10}{|c|}{ Progression and follow-up of patients with pathologically confirmed demyelinating disease } \\
\hline $\begin{array}{l}\text { EDSS at last follow-up, } \\
\text { median (IQR) }\end{array}$ & $3.0(1.5-4.0)$ & $3.0(1.5-4.0)$ & $3.0(2.0-3.5)$ & $3.0(1.5-4.0)$ & $3.0(1.8-3.5)$ & $1.0(0.0-2.0)$ & $3.0(1.8-4.0)$ & $2.0(1.0-3.8)$ & $3.0(2.0-4.0)$ \\
\hline $\begin{array}{l}\text { Years from disease } \\
\text { onset to antibody } \\
\text { testing, median (IQR) }\end{array}$ & $4.4(2.1-8.8)$ & $4.5(2.2-8.6)$ & $4.2(2.0-9.9)$ & $5.7(3.5-11.2)$ & $3.9(1.8-7.5)$ & $4.5(3.9-14.1)$ & $4.4(2.0-8.6)$ & $5.6(3.1-12.3)$ & $4.2(1.9-7.8)$ \\
\hline
\end{tabular}

${ }^{*}$ Immunomodulatory drugs include interferon- $\beta 1 \mathrm{a}$ and $1 \mathrm{~b}$ and glatiramer acetate. Immunosuppressant drugs include azathioprine, methotrexate, mitoxantrone and cyclophosphamide. Only one patient received acute steroids at the time of blood draw. Within six months of antibody testing, three patients received plasmapheresis. Acute 
Patients whose biopsies were sent in from other institutions occasionally lacked an indication for biopsy, but most underwent biopsy to exclude tumour. Some biopsies were performed to exclude vasculitis or infection. Clinical course and treatments were similar (all $P$-values $\geq 0.22$ ) for seropositive and negative MS patients tested by both Western blot and ELISA analysis (Table 1). Patients with IDS had similar frequencies of anti-MOG antibodies by both Western blot and ELISA, compared to patients with definite or probable MS $(P \geq 0.70)$ at last follow-up.

Patients seropositive for MOG IgG (by Western blot only) had a longer disease duration prior to antibody testing (median 5.7 versus 3.9 years, $P=$ 0.05) compared with seronegative patients. By ELISA, patients seropositive for MOG IgG had marginally longer disease duration prior to antibody testing (median 5.6 versus 4.2 years, $P=0.12$ ), although this relationship did not reach statistical significance. There was no correlation between disease duration prior to antibody testing for MOG IgM $(P>0.40)$. Disease severity (EDSS) was also similar for seropositive and negative patients $(P>0.10$, Table 1) for all tests except MOG IgM (ELISA only), where seropositive patients had a lower EDSS (median 1.0 versus $3.0, P=0.04$ ). However, only three patients were seropositive for MOG IgM by ELISA.

\section{Immunopathology, HLA DR status and MOG antibody status}

The median delay from the time of biopsy to time of blood draw (MOG antibody testing) was 3.1 years (IQR 1.4-7.1). Immunopathological classification revealed 12 pattern I, 43 pattern II and 17 pattern III. There were no pattern IV identified. Although 3 of 72 patients were seropositive for MOG IgM (ELISA) and all 3 had pattern II lesions, there were no statistically significant associations found between MOG antibody status by Western blot (Figure 2) or ELISA (Figure 3, Table 2) and IP.

There was a trend for an association between MOG IgM seropositivity (by Western blot only) and the presence of HLA DR2 $(P=0.09)$, but this relationship was not observed by ELISA testing (Table 3). As there were only six patients with two copies of the HLA DR2 allele, there was not sufficient power to assess the relationship of any of the antibodies tested and HLA DR2 heterozygosity or homozygosity.

\section{Plasmapheresis and MOG antibody status}

Nine patients received plasmapheresis during their clinical course for acute disabling attacks. MOG IgG or IgM seropositivity was not associated with plasmapheresis response. Antibody status as determined by Western blot or ELISA, response to treatment and associated treatments at time of antibody testing are presented in Table 4 .

\section{Correlation of Western blot and ELISA determined MOG antibody status}

\section{MOG $\lg G$}

Western blot analysis identified 19 seropositive and 53 seronegative patients. Of the 19 patients seropositive by Western blot, 13 were seronegative by ELISA and 10 of the 53 seronegative by Western blot were seropositive by ELISA. A low kappa (0.07) indicated very poor agreement.

\section{MOG IgM}

Western blot analysis identified 38 seropositive patients and 34 seronegative. Of the 38 patients identified as seropositive by Western blot, 35 were seronegative by ELISA and 0 of the 34 patients seronegative by Western blot were seropositive by ELISA. A low kappa (0.13) indicated very poor agreement.

\section{Stability over time $(n=4)$}

Serial blood draws at three-month intervals were collected in four RRMS patients beginning 9-22 months after their initial attack. No patients were seropositive for either IgM or IgG by ELISA. Fluctuations in antibody status in three of the four patients (as measured by Western blot) over time are shown in Figure 4. Patients 1 and 3 had no interval exacerbations and received no immunosuppressant medications (except for interferon- $\beta$ ) during the testing period. Patient 2 had several interval exacerbations and received steroid medications in addition to interferon- $\beta$ during the testing period. Patient 4 had a single exacerbation at the time of the third serial testing and was receiving chronic immunosuppressant therapy with mitoxantrone.

\section{Discussion}

The frequency of anti-MOG IgG in the biopsy MS cohort ( $26 \%$, by Western blot) is similar to that of non-biopsied MS patients (38\%) [4]. Prior studies have shown higher MOG antibody seropositivity by Western blot, compared to ELISA [3-5,25]. In our study, $22 \%$ of biopsied MS patients were positive for MOG IgG by ELISA, a similar frequency to that 


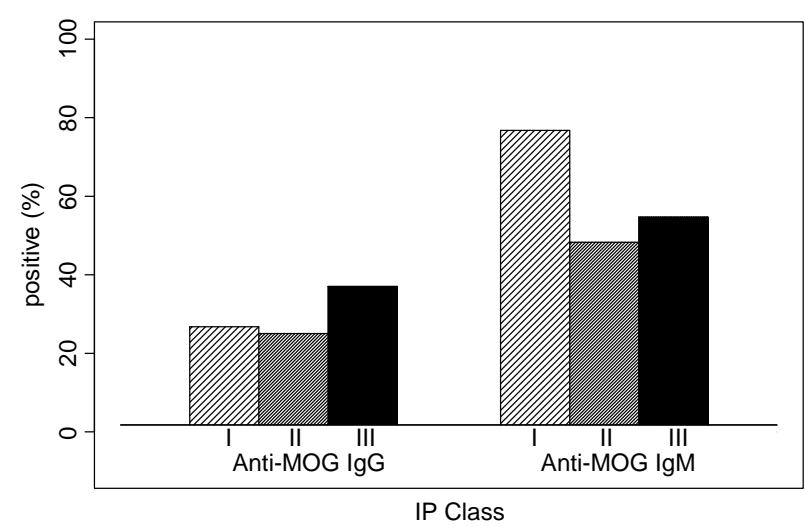

Figure 2 Western blot data for patterns I-III.
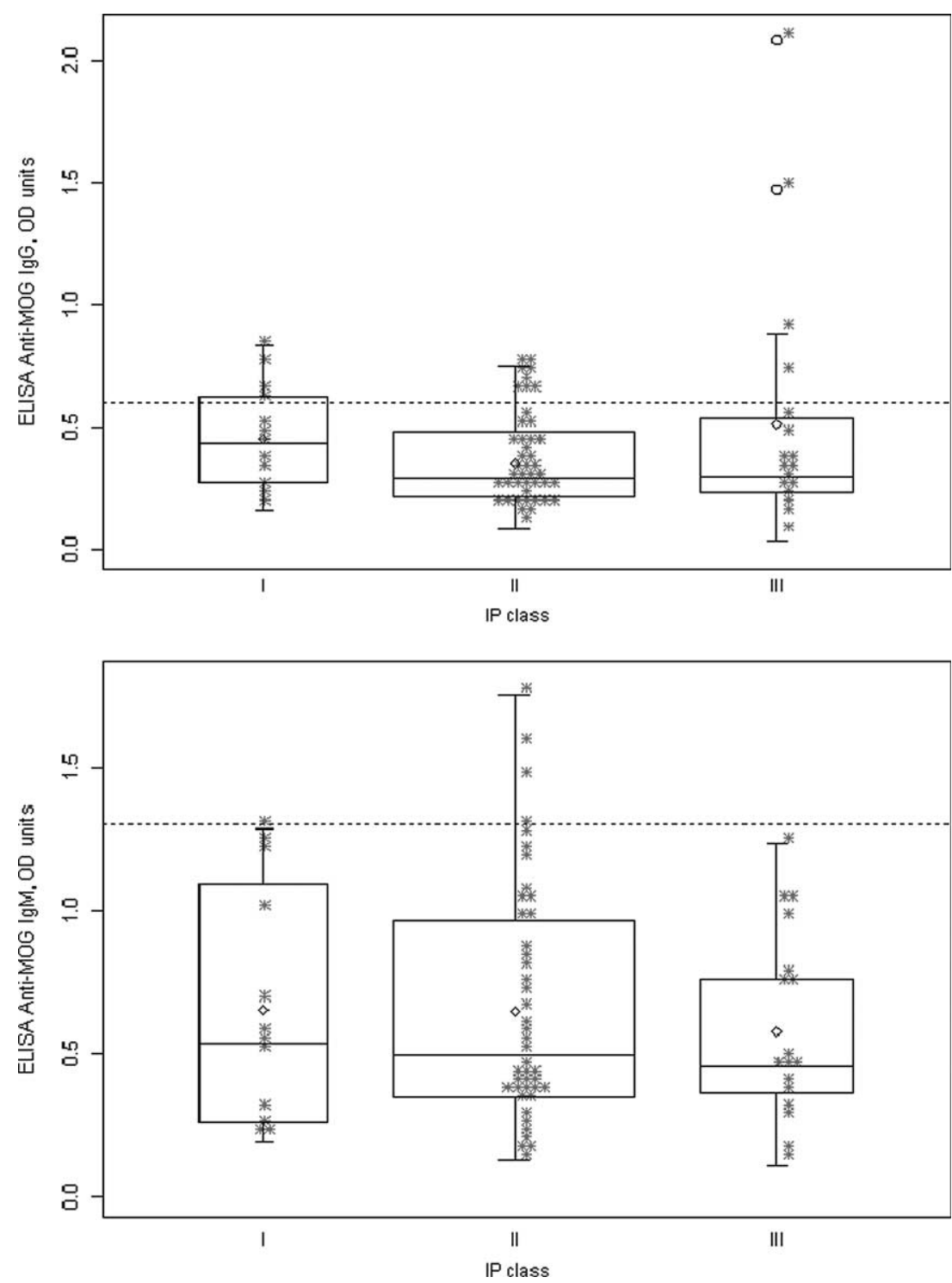

Figure 3 ELISA data for patterns I-III. 
Table 2 Antibody status of different IPs

\begin{tabular}{|c|c|c|c|c|c|}
\hline \multirow[b]{2}{*}{ Pattern type ( $n$, number of patients) } & \multicolumn{5}{|c|}{ Immunopathological classification } \\
\hline & Total $^{*}(n=72)$ & $\mathrm{I}(n=12)$ & II $(n=43)$ & III $(n=17)$ & $P$ value \\
\hline MOG IgM blot results (\%) & & & & & 0.23 \\
\hline - & $34(47)$ & $3(25)$ & $23(53)$ & $8(47)$ & \\
\hline+ & $38(53)$ & $9(75)$ & $20(47)$ & $9(53)$ & \\
\hline MOG IgG blot results (\%) & & & & & 0.61 \\
\hline- & $53(74)$ & $9(75)$ & $33(77)$ & $11(65)$ & \\
\hline+ & $19(26)$ & $3(25)$ & $10(23)$ & $6(35)$ & \\
\hline MOG IgM ELISA results (\%) & & & & & 0.74 \\
\hline$-(<1.3)$ & $69(96)$ & $12(100)$ & $40(93)$ & $17(100)$ & \\
\hline$+(>1.3)$ & $3(4)$ & $0(0)$ & $3(7)$ & $0(0)$ & \\
\hline MOG IgG ELISA results (\%) & & & & & 0.53 \\
\hline$-(<0.6)$ & $56(78)$ & $8(67)$ & $35(81)$ & $13(76)$ & \\
\hline$+(>0.6)$ & $16(22)$ & $4(33)$ & 8 (19) & $4(24)$ & \\
\hline
\end{tabular}

*72 patients had active lesions, which were classified into patterns I-III; no pattern IV were identified

reported in an Italian MS cohort (13.7\%), but a lower frequency than that obtained in Austrian MS patients (35\%) using the same methodology [10]. Lower frequencies of antimyelin antibodies have been reported by Lampasona et al., who found antiMOG IgG seropositivity in only $6 \%$ of MS patients using a liquid-phase radiobinding assay [9]. MOG IgM determined by Western blot was present in 53\% of our biopsied MS patients, a similar frequency to that reported for $261 \mathrm{MS}$ patients in Austria [5]. There was again a discrepancy between frequencies of MOG IgM seropositive patients by Western blot (53\%) compared with ELISA (4\%). This finding could reflect our recent observation that most of the MOG antibodies detected by immunoblot assays are directed against linear MOG epitopes, which are only partially exposed on the surface of myelin [26]. In contrast, anti-MOG antibodies measured by ELISA are directed against both linear and conformational epitopes [26].

The discrepancies between Western blot and ELISA antibody results, the different frequencies of MOG antibodies between studies and their high frequency in other neurological and non-neurological conditions raise questions regarding the sensitivity and specificity of this antibody for MS. Investigators cite technical differences in antigen preparation (denatured MOG for Western blot and soluble, refolded MOG for ELISA) and antibody determination as possible reasons for these discrepancies. This underscores the need for methodological consensus for the detection of these antibodies.

Recently Berger et al. suggested that MOG IgM antibodies (by Western blot) were predictors of development of MS in patients with clinically isolated syndromes [12]. However, in our study, patients who remained classified as IDS at last follow-up had similar frequencies of MOG antibodies (by Western blot) compared with patients that developed MS over a similar follow-up period. Thus, patients with pathologically proven demyelinating disease had a high frequency of anti-MOG antibodies (by Western blot) regardless of whether they developed MS or remained clinically isolated over a median of 4.4 years follow-up. As antibody testing

Table 3 Association of MOG antibodies and HLA DR 2 carrier status

\begin{tabular}{|c|c|c|c|c|}
\hline \multirow[b]{2}{*}{ Pattern type ( $n=$ number of patients) } & \multicolumn{4}{|c|}{ HLA DR 2 carrier } \\
\hline & Total $(n=69)$ & Yes $(n=37)$ & No $(n=32)$ & $P$ value \\
\hline MOG IgM blot results (\%) & & & & 0.09 \\
\hline 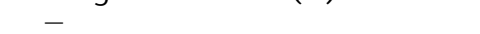 & 33 & $14(42)$ & $19(58)$ & \\
\hline+ & 36 & $23(64)$ & $13(36)$ & \\
\hline MOG IgG blot results (\%) & & & & 0.59 \\
\hline- & 50 & $28(56)$ & $22(44)$ & \\
\hline+ & 19 & $9(47)$ & $10(53)$ & \\
\hline MOG IgM ELISA results (\%) & & & & 0.24 \\
\hline$-(<1.3)$ & 66 & $34(52)$ & $32(48)$ & \\
\hline$+(>1.3)$ & 3 & $3(100)$ & $0(0)$ & \\
\hline MOG IgG ELISA results (\%) & & & & 0.57 \\
\hline$-(<0.6)$ & 53 & $27(51)$ & $26(49)$ & \\
\hline$+(0.6)$ & 16 & $10(62)$ & $6(38)$ & \\
\hline
\end{tabular}


Table 4 Clinical and serological characteristics of patients treated with plasmapheresis for steroid-resistant disabling exacerbations

\begin{tabular}{|c|c|c|c|c|c|c|c|c|}
\hline & \multirow{2}{*}{$\begin{array}{l}\text { Years from } \\
\text { PLEX to } A b \text { test }\end{array}$} & \multirow{2}{*}{$\begin{array}{l}\text { Significant } \\
\text { improvement }\end{array}$} & \multirow{2}{*}{$\begin{array}{l}A B C \text { drugs at } \\
\text { time of } A b \text { test }\end{array}$} & \multirow{2}{*}{$\begin{array}{l}\text { Received } \\
\text { immunosuppressant } \\
\text { within } 6 \text { months of } A b \text { test }\end{array}$} & \multicolumn{2}{|c|}{ Western blot } & \multicolumn{2}{|c|}{ ELISA } \\
\hline & & & & & MOG IgG & MOG IgM & MOG IgG & MOG IgM \\
\hline 1 & 7.2 & Success & - & - & - & - & - & - \\
\hline 2 & 5.3 & Success & + & - & - & - & - & - \\
\hline 3 & 13.5 & Success & + & + & + & + & + & - \\
\hline 4 & 4.2 & Success & + & - & + & - & - & - \\
\hline 5 & 2.1 & Success & - & - & - & - & + & - \\
\hline 6 & 5.2 & Success & + & - & - & - & - & - \\
\hline 7 & 0.1 & Failure & + & - & - & - & - & - \\
\hline 8 & 1.8 & Failure & + & - & + & + & - & - \\
\hline 9 & 1.9 & Failure & - & - & - & + & - & - \\
\hline
\end{tabular}

Plasmapheresis PLEX; ABC drugs, immunomodulatory drugs include interferon- $\beta$ 1a and $b$ and glatiramer acetate; Ab, antibody

was performed a median 4.4 years after disease onset, this group of patients differ from those described by Berger et al., in whom MOG antibody status was analysed at disease presentation following a clinically isolated syndrome in the setting of MRI dissemination. Thus the role of MOG antibodies as a prognostic marker following disease presentation cannot be addressed in this study. Nonetheless, a recent study on a CIS cohort with samples taken at baseline also failed to detect an association of anti-MOG antibodies with conversion to CDMS [13].

In this study, MOG IgG (by both methodologies) and MOG IgM (by Western blot) were not related to disease severity or clinical course. Although the three patients seropositive for MOG IgM (by ELISA) had a lower EDSS than seronegative patients, it is not possible to draw conclusions given the low numbers of seropositive patients and the multiple statistical comparisons. Patients with a longer duration of disease at the time of antibody testing were more likely to have anti-MOG IgG, although this was not the case for anti-MOG IgM. Thus patients may tend to convert from seronegative to
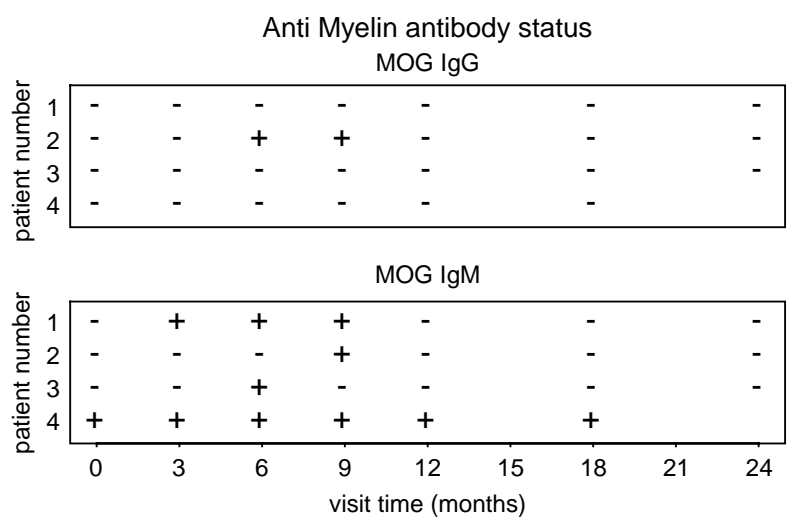

Figure 4 Anti-MOG antibody status (by Western blot) on serial evaluations in four RRMS patients. seropositive for MOG IgG as disease duration increases.

Despite reports of MOG IgG inducing demyelination in T-cell-mediated experimental autoimmune encephalomyelitis (EAE), and the identification of autoantibodies against myelin in the marmoset model of EAE and acute MS lesions, their pathogenic role in MS is uncertain $[27,28]$. Experimental studies have shown that the selection for an antibody response against the demyelinating conformational MOG epitope is under genetic control of the MHC region [7]. This control appears to be mediated not by the HLA-antigen themselves, but by proteins that are homologous to MOG and are encoded within the MHC. In this study we found no association between HLA DR-2 status and MOG antibodies measured by either Western blot or ELISA. Although there is profound linkage disequilibrium within the MHC region, testing for HLA-DR genotype may be insufficient to detect a genetic influence on MOG antibody reactivity in a small sample of patients, as was the case in this study.

Our study also did not find any association between antibody status and IP, arguing against a pathogenic role in MS for the autoantibodies to bacterially produced human recombinant MOG measured in this study. Although all three patients seropositive for MOG IgM by ELISA had pattern II lesions, this was not statistically significant (though likely underpowered). The absence of an association in this study between MOG antibodies recognizing linear MOG epitopes (measured by Western blot) with MS IPs may in part be explained by their limited ability to induce demyelination in vivo $[6,29]$. The anti-MOG antibodies measured by ELISA in this study are directed against soluble, refolded MOG produced in Escherichia coli, which lacks the correct membrane topology and glycosylation. These factors are considered crucial for a pathogenic role of MOG antibodies [8,30,31]. Furthermore, only a small fraction of anti-MOG antibodies are directed against a conformational epitope of this 
molecule and can mediate demyelination in vivo and in vitro $[6,29,32,33]$. A recent study revealed that only anti-MOG antibodies that are found in MS lesions have high affinity to their antigen, whereas serum and CSF antibodies to MOG are low-affinity antibodies [11]. It will be important for future studies to analyse these particular MOG antibodies in relation to the patterns of demyelination.

Keegan et al. recently showed that patients with MS with pattern II pathology are more likely to respond favourably to TPE than are patients with patterns I or III [17]. Few reports exist regarding myelin antibody status and response to therapy. One small study suggested that patients with acute severe attacks of other CNS demyelinating diseases (eg, neuromyelitis optica, Marburg variant and acute transverse myelitis) may be more commonly seropositive for anti-MOG IgG than MS patients. A trend in that study suggested that patients with MOG IgG seropositivity may be more likely to respond favourably to TPE than seronegative patients [34]. In this study, we found no association between MOG antibody status and response to TPE for an acute severe attack of MS, though any meaningful interpretation of the data is limited by the small sample size and the delay between TPE and MOG antibody testing (median of 4.2 years).

A further limitation of this study is the delay between time of biopsy and blood draw for antibody testing (median 3.1 years). In addition, the fluctuations in MOG antibody status measured by Western blot in three of four RRMS patients with serial testing raise questions regarding the stability of this biomarker. These fluctuations could not be explained based on concomitant use of immunosuppressive or immunomodulatory drugs. Nonetheless, the effect of immunomodulatory or immunosuppressant medications and clinical and subclinical disease activity on antibody status needs consideration when making clinicopathological and serological correlations.

The lack of agreement between Western Blot and ELISA methodologies underscores the need for methodological consensus for anti-MOG antibody measurement. Recent results of a first multicentre validation of methods used for anti-MOG antibody measurement have shown inconsistent results, as was the case in the present study (M Reindl and $\mathrm{T}$ Berger, unpublished). Until there is a consensus on what exactly is being measured and the optimal methodology to use (ELISA or Western blot), it will be difficult to make any meaningful interpretation of the pathophysiological, clinical or predictive relevance of these antibodies in MS.

The results of this study clearly indicate that the antigen and methods used were not reliable to detect anti-MOG antibodies correlating with MS pathology. Therefore optimized methods using correctly folded and glycosylated human MOG as an antigen have to be developed for the measurement of these antibodies. Recent studies suggest that increased serum levels of antibodies to correctly folded and glycosylated MOG could indeed be detected in MS patients using mammalian cells expressing human MOG on their surface $[8,35]$. Moreover, another recent study indicated the importance of antibodies to oligodendrocyte surface antigens in MS [36]. Other candidate targets on the myelin surface also need to be considered, including the recently reported aquaporin 4 water channel protein associated with neuromyelitis optica $[37,38]$.

As the immunopathological characteristics and reported preferential plasmapheresis response associated with pattern II lesions suggest a pathogenic role for antibody-mediated demyelination in a subset of MS patients, it will be important to measure these in our MS biopsy cohort.

\section{Acknowledgements}

We thank Mary Bennett for secretarial assistance, Patricia Ziemer, Carolyn Rainer and Kathrin Schanda for technical support, and Linda Linbo for nursing support. This work was supported by the National Multiple Sclerosis Society (RG-3185-A-2 to CFL) and by M01 RR00585, General Clinical Research Centers Program.

\section{References}

1. Xiao BG, Linington C, Link $\mathbf{H}$. Antibodies to myelinoligodendrocyte glycoprotein in cerebrospinal fluid from patients with multiple sclerosis and controls. J Neuroimmunol 1991; 31: 91-96.

2. Karni A, Bakimer-Kleiner R, Abramsky o, BenNun A. Elevated levels of antibody to myelin oligodendrocyte glycoprotein is not specific for patients with multiple sclerosis. Arch Neurol 1999; 56: 311-15.

3. Lindert RB, Haase CG, Brehm $U$, Linington $C$, Wekerle H, Holfeld R. Multiple sclerosis: B- and Tcell responses to the extracellular domain of the myelin oligodendrocyte glycoprotein. Brain 1999; 122: $2089-100$

4. Reindl M, Linington C, Brehm U, Egg R, Dilitz E, Deisenhammer $\mathbf{F}$ et al. Antibodies against the myelin oligodendrocyte glycoprotein and the myelin basic protein in multiple sclerosis and other neurological diseases: a comparative study. Brain 1999; 122: 2047-56.

5. Egg R, Reindl M, Deisenhammer $\mathbf{F}$, Linington $C$, Berger T. Anti-MOG and anti-MBP antibody subclasses in multiple sclerosis. Mult Scler 2001; 7: 285-89.

6. Haase CG, Guggenmos $\mathbf{J}$, Brehm $\mathbf{U}$, Andersson $M$, Olsson T, Reindl $\mathbf{M}$ et al. The fine specificity of the myelin oligodendrocyte glycoprotein autoantibody response in patients with multiple sclerosis and normal healthy controls. J Neuroimmunol 2001; 114: 220-25.

7. Lutterotti A, Reindl $M$, Gassner C, Poustka $K$, Schanda K, Deisenhammer F et al . Antibody response 
to myelin oligodendrocyte glycoprotein and myelin basic protein depend on familial background and are partially associated with human leukocyte antigen alleles in multiplex families and sporadic multiple sclerosis. J Neuroimmunol 2002; 131: 201-207.

8. Gaertner S, de Graaf KL, Greve B, Weissert $R$. Antibodies against glycosylated native MOG are elevated in patients with multiple sclerosis. Neurology 2004; 63: $2381-83$.

9. Lampasona V, Fanciotta D, Furlan R, Zanaboni S, Fazio R, Bonifacio $\mathbf{E}$ et al. Similar low frequency of anti-MOG IgG and IgM in MS patients and healthy subjects. Neurology 2004; 62: 2092-94.

10. Mantegazza R, Cristaldini P, Bernasconi P, Baggi F, Pedotti R, Piccini I et al. Anti-MOG autoantibodies in Italian multiple sclerosis patients: specificity, sensitivity and clinical association. Int Immunol 2004; 16: 559-65.

11. O'Connor KC, Appel H, Bregoli L, Call ME, Catz I, Chan JA et al . Antibodies from inflamed central nervous system tissue recognizes myelin oligodendrocyte glycoprotein. I Immunol 2005; 175: 1974-82.

12. Berger T, Rubner P, Schautzer F, Egg R, Ulmer H, Mayringer I et al. Anti-myelin antibodies as a predictor of clinically definite multiple sclerosis after a first demyelinating event. $N$ Engl J Med 2003; 349: 139-45.

13. Lim ET, Berger T, Reindl M, Dalton CM, Fernando $\mathbf{K}$, Keir G et al. Anti-myelin antibodies do not allow earlier diagnosis of multiple sclerosis. Mult Scler 2005; 11: 492-94.

14. Lucchinetti CF, Brüick W, Rodriguez M, Lassmann H. Distinct patterns of multiple sclerosis pathology indicates heterogeneity on pathogenesis. Brain Pathol 1996; 6: 259-74.

15. Lucchinetti C, Brück W, Parisi J, Scheithauer B, Rodriguez M, Lassmann H. Heterogeneity of multiple sclerosis lesions: implications for the pathogenesis of demyelination. Ann Neurol 2000; 47: 707-17.

16. Lucchinetti CF, Bruck W, Lassmann H. Evidence for pathogenic heterogeneity in multiple sclerosis. Ann Neurol 2004; 56: 308.

17. Keegan M, Konig F, McClelland R, Bruck $\mathbf{W}$, Morales Y, Bitsch A et al. Relation between humoral pathological changes in multiple sclerosis and response to therapeutic plasma exchange. Lancet 2005; 366: $579-82$.

18. Lassmann H, Brück W, Lucchinetti C. Heterogeneity of multiple sclerosis pathogenesis: implications for diagnosis and therapy. Trends Mol Med 2001; 7: 115-21.

19. Hart MN, Earle KM. Haemorrhagic and perivenous encephalitis: a clinical-pathological review of 38 cases. J Neurol Neurosurg Psychiatry 1975; 38: 585-91.

20. Linares D, Echevarria I, Mana P. Single-step purification and refolding of recombinant mouse and human myelin oligodendrocyte glycoprotein and induction of EAE in mice. Protein Expr Purif 2004; 34: 249-56.

21. Olerup o, Zetterquist H. HLA-DR typing by PCR amplification with sequence specific primers (PCR-SSP) in 2 hours: an alternative to serological DR typing in clinical practice including donor-recipient matching in cadaveric transplantations. Tissue Antigens 1992; 39: 225-35.

22. Kurtzke JF. Rating neurologic impairment in multiple sclerosis: an expanded disability status scale (EDSS). Neurology 1983; 33: 1444-52.

23. Poser CM, Paty DW, Scheinberg L, McDonald WI, Davis FA, Ebers GC et al. New diagnostic criteria for multiple sclerosis: guidelines for research protocols. Ann Neurol 1983; 13: 227-31.
24. McDonald WI, Compston A, Edan G, Goodkin D, Hartung, HP, Ludwin FD et al. Recommended diagnostic criteria for multiple sclerosis: guidelines from the International Panel on the diagnosis of multiple sclerosis. Ann Neurol 2001; 50: $121-27$.

25. Kennel De March A, De Bouwerie M, Kolopp-Sarda MN, Faure GC, Bene MC, Bernard CC et al. Antimyelin oligodendrocyte glycoprotein B-cell responses in multiple sclerosis. J Neuroimmunol 2003; 135: 117-25.

26. Khalil M, Reindl M, Lutterotti A, Kuenz B, Ehling $\mathbf{R}$, Gneiss $\mathbf{C}$ et al. Epitope specificity of serum antibodies directed against the extracellular domain of myelin oligodendrocyte glycoprotein: influence of relapses and immunomodulatory treatments. J Neuroimmunol 2006; 174: $147-56$

27. Genain CP, Cannella B, Hauser SL, Raine CS Identification of autoantibodies associated with myelin damage in multiple sclerosis. Nat Med 1999; 5: 170-75.

28. Schluesener HJ, Sobel RA, Linington C, Weiner HL. A monoclonal antibody against a myelin oligodendrocyte glycoprotein induces relapses and demyelination in central nervous system autoimmune disease. I Immunol 1987; 139: 4016-21.

29. Bourquin C, Schubart A, Tobollik S, Mather I, Ogg $\mathbf{S}$, Liblau $\mathbf{R}$ et al. Selective unresponsiveness to conformational B cell epitopes of the myelin oligodendrocyte glycoprotein in $\mathrm{H}-2 \mathrm{~b}$ mice. I Immunol 2003; 171: 455-61.

30. Marta CB, Oliver AR, Sweet RA, Pfeiffer FC, Ruddle NH. Pathogenic myelin oligodendrocyte glycoprotein antibodies recognize glycosylated epitopes and perturb oligodendrocyte physiology. Proc Natl Acad Sci U S A 2005; 102: 13992-97.

31. Lolli F, Mulinacci B, Carotenuto A, Bonetti B, Sabatino G, Mazzanti B et al. An N-glucosylated peptide detecting disease-specific autoantibodies, biomarkers of multiple sclerosis. Proc Natl Acad Sci U S A 2005; 102: 10273-78.

32. von Budingen HC, Hauser SL, Ouallet JC, Tanuma M, Menge G, Genain CP. Frontline: epitope recognition on the myelin/oligodendrocyte glycoprotein differentially influences disease phenotype and antibody effector functions in autoimmune demyelination. Eur $J$ Immunol 2004; 34: 2072-83.

33. Mathey E, Breithaupt C, Schubart AS, Linington C. Commentary: sorting the wheat from the chaff: identifying demyelinating components of the myelin oligodendrocyte glycoprotein (MOG)-specific autoantibody repertoire. Eur I Immunol 2004; 34: 2065-71.

34. Keegan $M$, Berger $T$, Lucchinetti $\mathbf{C}$, Reindl $M$, Rodriguez $\mathbf{M}$, Noseworthy $\mathbf{J H}$ et al. Antibodies to myelin proteins in patients with severe attacks of demyelinating disease who undergo therapeutic plasma exchange. Neurology 2001; 56: A366.

35. Lalive PH, Menge T, Delarasse C, Della Gaspera B, Pham-Dinh D, Villoslada $\mathbf{P}$ et al . Antibodies to native myelin oligodendrocyte glycoprotein are serologic markers of early inflammation in multiple sclerosis. Proc Natl Acad Sci U S A 2006; 103: 2280-85.

36. Lily O, Palace J, Vincent A. Serum autoantibodies to cell surface determinants in multiple sclerosis: a flow cytometric study. Brain 2004; 127: 269-79.

37. Trotter J. NG2-positive cells in CNS function and the pathological role of antibodies against NG2 in demyelinating diseases. J Neurol Sci 2005; 233: 37-42.

38. Lennon VA, Kryzer T, Pittock SJ, Verkman AS, Hinson SR. IgG marker of optic-spinal multiple sclerosis binds to the aquaporin-4 water channel. J Exp Med 2005; 202: $473-77$. 